\title{
Myxoide Pseudozysten - Diagnose und Therapie
}

\author{
Myxoide Pseudocyst - Diagnosis and Therapy
}

Autoren

Institute

\section{O. Mainusch ${ }^{1}$, V. Mielke², C. Schulte}

Facharztpraxis für Dermatologie und Allergologie, Velbert, Heiligenhaus

Fachübergreifende Gemeinschaftspraxis für Dermatologie und Pathologie (M. Reusch, U. Reusch, E. Christophers und V. Mielke), Hamburg

\section{Bibliografie}

DOI $10.1055 / \mathrm{s}-2007-966311$

Akt Dermatol 2007; 33 :

82-86 ๑ Georg Thieme

Verlag KG Stuttgart · New York ISSN 0340-2541

Korrespondenzadresse

Dr. Oliver Mainusch

Blumenstr. 1

42551 Velbert

dr.derm@web.de

\section{Zusammenfassung \\ $\nabla$}

Die von der Gelenkkapsel ausgehende ganglionartige Ausstülpung, die zur Verformung des Nagelbetts oder Nagels führt, wird als Klarzyste, Mukozyste, myxoide Zyste oder Dorsalzyste bezeichnet. Ihr Druck auf die germinale Matrix

\section{Einleitung}

Myxoide Pseudozysten sind benigne pseudotumoröse Veränderungen, die gewöhnlich im proximalen Nagelwall entstehen. Ätiologie und Pathogenese sind bis heute ungeklärt. Das ist auch der Grund, warum die myxoiden Pseudozysten unter zahlreichen Synonymen wie dorsale Fingerzyste, Dorsalzyste, Synovialzyste, rezidivierende myxoide Zyste, dorsales digitales Gelenksganglion, digitale muzinöse Pseudozyste oder myxomatöse Degeneration aufgeführt werden [9]. Unklar ist ebenso, ob es verschiedene Typen der myxoiden Pseudozysten gibt. Möglicherweise aber beruhen die myxoiden Pseudozysten auf einer fokalen Degeneration des Bindegewebes ohne typische Auskleidung mit Synovialis. Dieser Degeneration können möglicherweise aktinische Schädigungen oder wiederholt geringe Traumen zugrunde liegen. An der geschädigten Stelle kommt es dann zu einem locus minoris resistentiae mit folgender Hernisation des distalen Interphalangealgelenkes.

Myxoide Pseudozysten finden sich häufiger bei Frauen als bei Männern. Sie kommen zehnmal häufiger an den Fingern als an den Zehen vor. Zeige-, Mittel- und Ringfinger weisen am häufigsten myxoide Pseudozysten auf. Sie beginnen als schmerzlose kleine Schwellung, die langsam bis auf Erbsengröße anwachsen. Die Konsistenz variiert von weich bis fest, von homogen bis fluktuierend. Die Oberfläche ist glatt, oft ohne Veränderung des normalen Hautoberflächenreliefs, sel- führt zur Nageldeformität. Wächst die Zyste oberhalb des Nagelwalls, kommt es zu einer Grube in der Nagelplatte. Dehnt sich die Zyste zwischen Periost und germinaler Matrix aus, wird die Nagelplatte zerstört. Eine chirurgische Behandlung sollte erfolgen.

ten können sie einmal verrukös oder gar ulzeriert sein. Diaphanoskopisch weist sie deutlich eine zystische Struktur auf. Wegen der Strecksehnen sind sie immer nur auf einer Seite der Mittellinie lokalisiert. Nebenbefundlich finden sich meist auch Heberden-Knötchen und eine degenerative Osteoarthritis des Endgelenkes.

Es handelt sich, bedingt durch ihr langsames Wachstum, um eine chronische, oft Jahre vor ihrer Diagnose bestehende Veränderung. Je nach Lokalisation führt sie zu Veränderungen der Nagelplatte. Ihr häufigster Sitz, am proximalen Nagelwall, führt charakteristischerweise zu einer longitudinalen Einsenkung der Nagelplatte. Eine Ruptur der Zyste in den Raum unter den proximalen Nagelwall mit Entleerung einer geruchlosen, farblosen, gelatinösen viskösen Substanz ist häufig und führt zu wellenartigen Unregelmäßigkeiten mit längsverlaufenden Einsenkungen der Nagelplatte. Wiederholt erfolgloses Operieren kann zu einer Verlagerung der myxoiden Pseudozyste unter die Nagelmatrix (sekundäre subunguale myxoide Pseudozysten) führen. Primäre subunguale myxoide Pseudozysten sind erst in jüngster Zeit beschrieben worden.

Zur Therapie der myxoiden Pseudozysten gibt es bisher keine einheitlichen Therapieempfehlungen. Die Behandlungsverfahren sind so häufig wie es Synonyme für myxoide Pseudozysten gibt. Die Therapie der Wahl mit der geringsten Rezidivrate stellt die operative Pseudozystenentfernung dar. 
Abhängig von der Lage der Zyste finden sich verschiedene Nageldeformitäten bis hin zur vollständigen Nageldestruktion. Aufgrund von Lage und Größe der Zyste sollte der chirurgische Eingriff geplant werden. Häufig sind hierzu kleinere Lappenplastiken (Mini-Flaps) erforderlich, da lokalisationsbedingt direkte Primärverschlüsse unmöglich werden.

Im Folgenden wollen wir verschiedene Zystenlokalisationen aufzeigen und deren operative Therapie anhand eines klinischen Falles vorstellen.

\section{Anamnese}

$\nabla$

Seit längerer Zeit habe der 52-jährige Patient am rechten Mittelfinger einen mehrhöckerigen Knoten bemerkt, der langsam größer geworden sei. Zuletzt sei eine Rillenbildung des Fingernagels aufgefallen, die sich vom proximalen Nagelwall aus auf die gesamte Nagelplatte ausgedehnt habe. Schmerzen bestünden nur bei gelegentlicher Berührung. Der Knoten sei aber bei manuellen Tätigkeiten hinderlich.

\section{Befund}

$\nabla$

Am rechten Mittelfingerendglied bis zur proximalen Nagelfalz ziehend zeigt sich dorsal eine $1 \mathrm{~cm}$ große, prall elastische, zweikammerige, bläulich schimmernde zystische Schwellung mit Krustenauflagerung und positiver Diaphanoskopie, in deren Verlängerung nach distal die Nagelplatte leicht kanalförmig eingesunken ist ( $\bullet$ Abb. 1). Da die gesamte Nagelplatte von der Rille durchzogen wird, liegt die zeitliche Entstehung mehr als ein halbes Jahr zurück.

\section{Therapie \\ $\nabla$}

Nach klinischer und diaphanoskopischer Untersuchung wirde in Leitungsanästhesie nach Oberst die Exstirpation der myxoiden Pseudozyste durchgeführt. Die Operation erfolgt in Blutleere. Hierzu setzen wir einen normalen sterilen Operationshandschuh ein, der am Ende des betroffenen Fingers eingeschnitten und aufgerollt wird. Zur präoperativen Darstellung der Pseudozyste mit ihrem Stiel injizieren wir 0,05-0,1 ml Methylenblaulösung intraartikulär von volar ( $\odot$ Abb. 2 ) in das distale Interphalangealgelenk. Die vorher glasig schimmernde Pseudozyste färbt sich schnell blau an. Die über der Zyste liegende Haut wird rund umschnitten und abpräpariert. Der „Stiel“ der Pseudozyste, durch die Methylenblaudarstellung gut zu verfolgen, wird mittels resorbierbarem Nahtmaterial ligiert. Zur Rezidivprophylaxe sollte der ligierte Stiel noch mit einer dünnen Hautweichteilschicht bedeckt werden. Anschließend wird die gelöste Haut wieder zurückgeklappt und vernäht ( $\odot$ Abb.3- $\odot 9$ ).

Je nach Lokalisation der Zyste und Zustand der bedeckenden Haut ist ein direkter Primärverschluss möglich, oder es muss auf kleine lokale, gut durchzuführende Lappenplastiken zurückgegriffen werden ( $\odot$ Abb.10- $\bigcirc$ 14). Sowohl bei direktem Primärverschluss als auch bei lokalen Mini-Flaps gestaltet sich der postoperative Heilungsverlauf in der Regel komplikationslos. Bei rupturierten oder infizierten Zysten empfiehlt sich der perioperative Einsatz von Antibiotika. Es hat sich z.B. Clindamycin $3 \times 600 \mathrm{mg}$ pro Tag bei oraler Gabe bewährt. Vor dem Verbands-

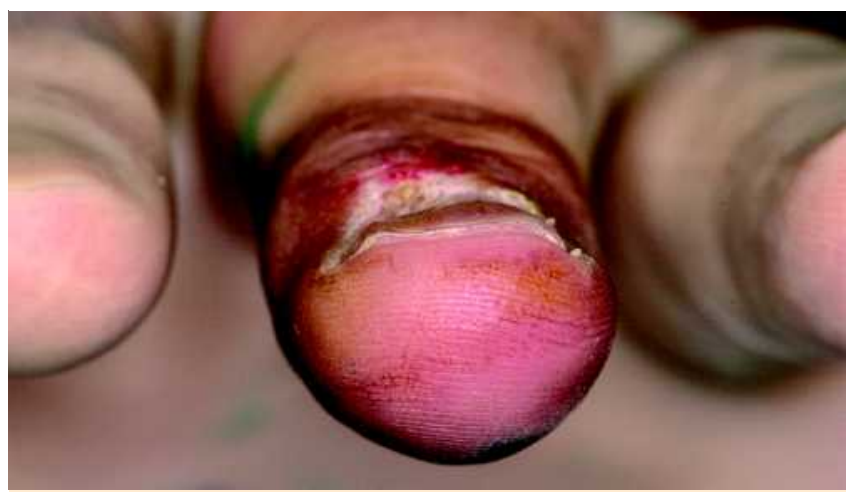

Abb. 1 Klinisches Bild einer myxoiden Pseudozyste am Finger.

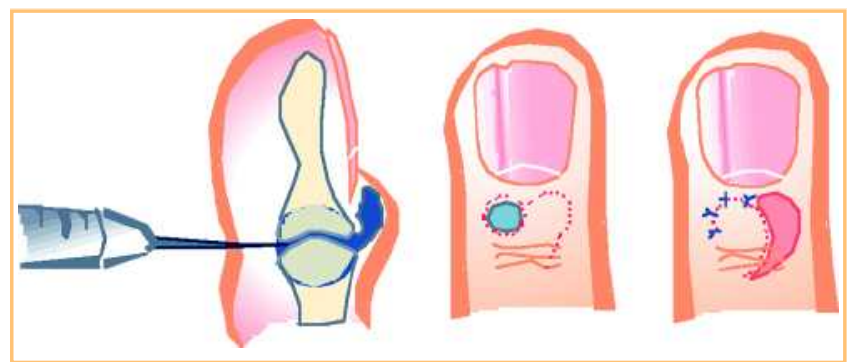

Abb. 2 Anfärben der myxoiden Pseudozyste im Gelenkspalt mit Methylenblau, mögliches operatives Vorgehen bei Lokalisation am proximalen Nagelwall.

wechsel empfehlen sich chirurgische Fingerbäder mit einem desinfizierenden Zusatz.

\section{Histologie \\ $\nabla$}

Das Exzidat zeigt innerhalb der oberen und mittleren Dermis einen nicht von einem Epithel ausgekleideten pseudozystischen Hohlraum mit leichter Fibrose im Randbereich. Es stellt sich im Wandbereich keine Synovialis und keine Endothelauskleidung dar. Im Lumen lässt sich diffus reichlich muzinöses Material nachweisen (Alcianblaufärbung). Obwohl die Kollagenfasern am Rand leicht komprimiert erscheinen, bilden sie keine echte Kapsel. Oft findet sich auch in der Umgebung in geringem Umfang eine muzinöse basophile Substanz, die Hinweise auf die Genese derartiger Pseudozysten liefern könnte. Diagnose: Myxoide Pseudozyste ( $\odot$ Abb. 15 und $\odot$ 16).

\section{Diskussion}

$\nabla$

Bei der myxoiden Pseudozyste des Fingers, auch dorsale Fingerzyste oder mukoide Dorsalzyste genannt, handelt es sich um einen ganglionartigen Hohlraum, dessen Ätiologie bisher ungeklärt ist, meist aber bei Patienten mit degenerativen Fingerendgelenksveränderungen oder nach traumatischen Gelenkaffektionen zu finden ist. Seltener können auch einmal Zehenendglieder betroffen sein [10] ( $\bullet$ Abb. 17). Eine Verbindung zu dem distalen Interphalangealgelenk kann durch intraartikuläre Injektion von kontrastierenden Farbstoffen, z. B. Methylenblau, Patentblau oder durch bildgebende Verfahren wie Kernspintomografie oder Arthrografie gezeigt werden [2]. Bei der Kern- 


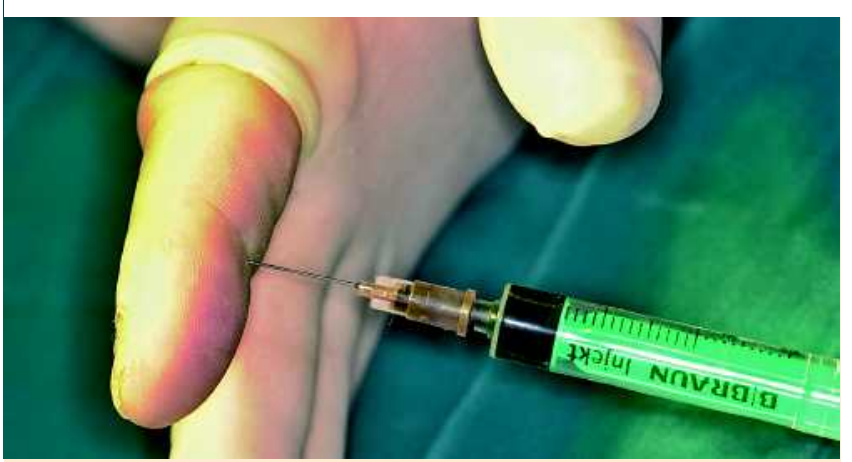

Abb. 3 Anfärben der Pseudozyste mit Methylenblau.

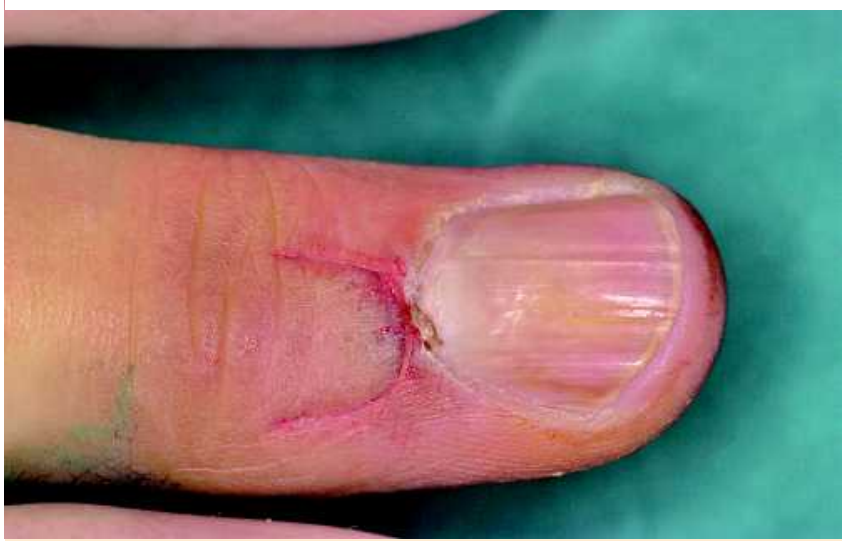

Abb. 5 Umschneiden des Lappens.

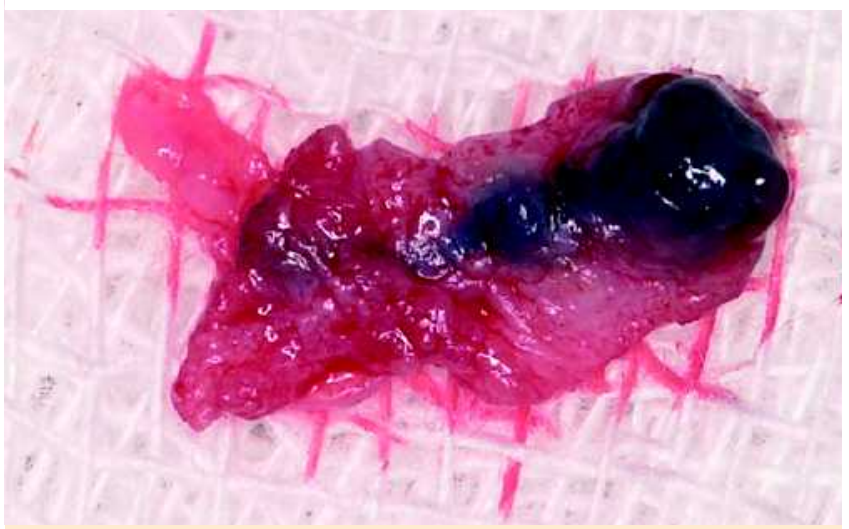

Abb. 7 Präparat.

spintomografie empfiehlt es sich, den Finger vor der Untersuchung dick mit Vaseline zu bestreichen. So lassen sich die Strukturen und die Haut besser abgrenzen. Wie der Name mukoide Dorsalzyste besagt, finden sich bläulich durchschimmernde Knötchen meist an den streckseitigen Fingerendgliedern nahe der Nagelfalz. In paraungualer oder sogar subungualer Lokalisation finden sie sich seltener primär, häufiger jedoch nach fehlgeschlagener Behandlung oder als Rezidiv ( $\bullet$ Abb. 17 und $\bullet$ 18). Je nach Beziehung zur Nagelmatrix kann die Pseudozyste eine kanalförmige Dystrophie des Nagels (Spaltnagelbildung) verursachen oder sogar zur Onychodystrophie führen. Subunguales Wachstum verursacht starke Druck- und Bewegungsschmerzen, ähnlich einem Hämatom oder Glomustumor. Differenzialdiagnostisch ist an das Onychomatrikom, das subunguale eruptive

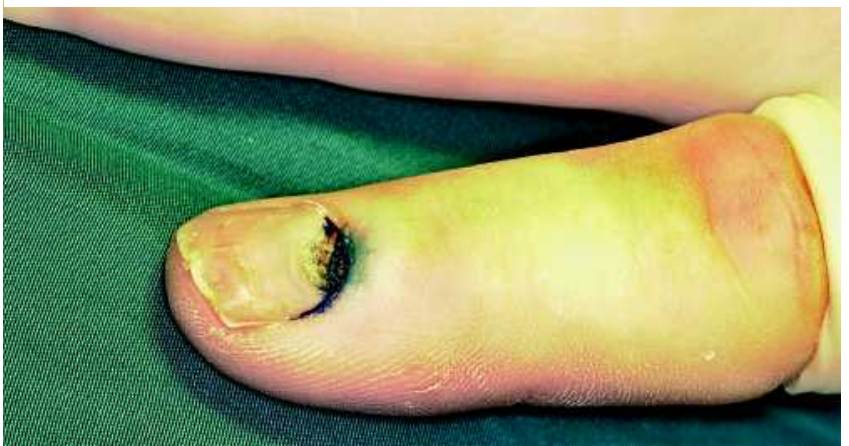

Abb. 4 Angefärbte Pseudozyste.

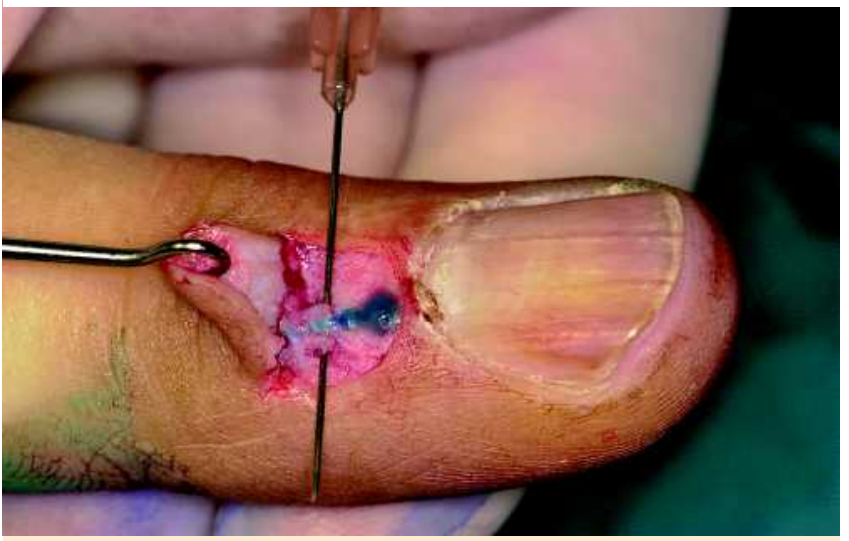

Abb. 6 Darstellung der Pseudozyste mit Stiel nach Anheben der bedeckenden Haut.

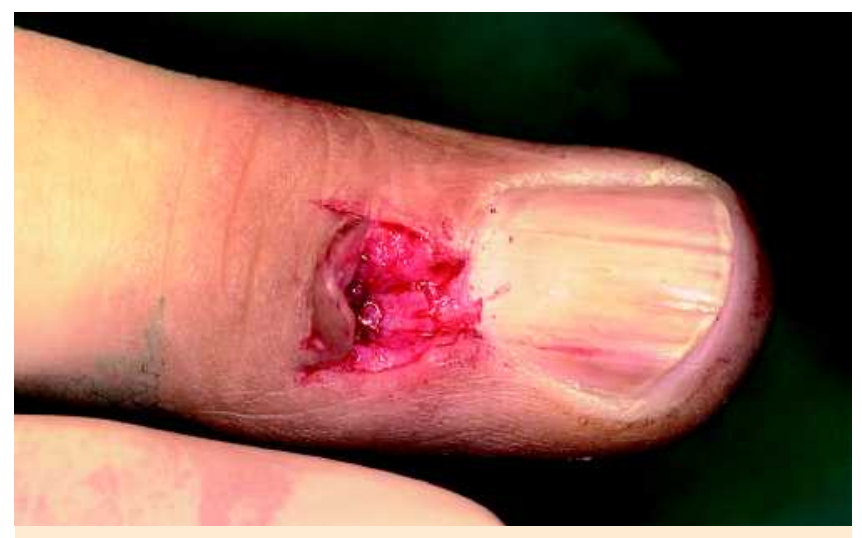

Abb. 8 Leere Defekthöhle.

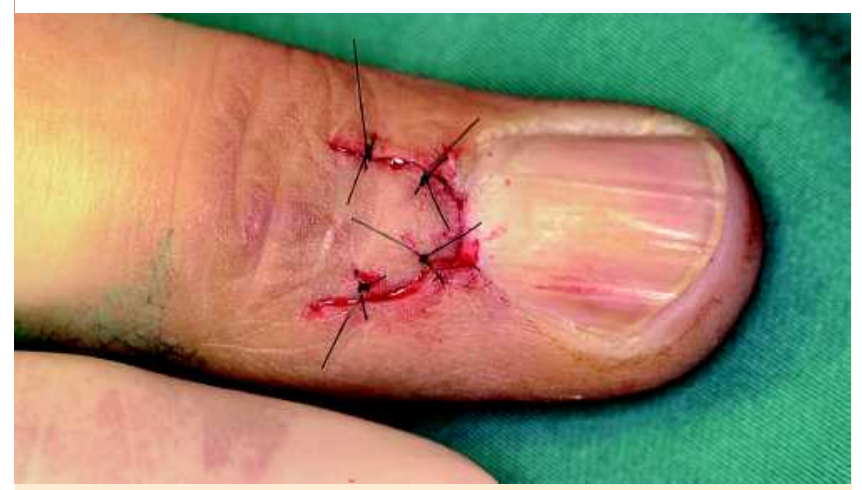

Abb. 9 Verschluss. 


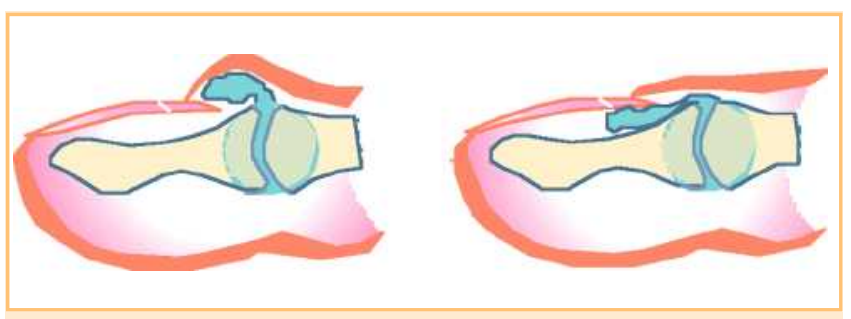

Abb. 10 Typische Lagen der myxoiden Pseudozysten mit Verbindung zum Interphalangialraum.

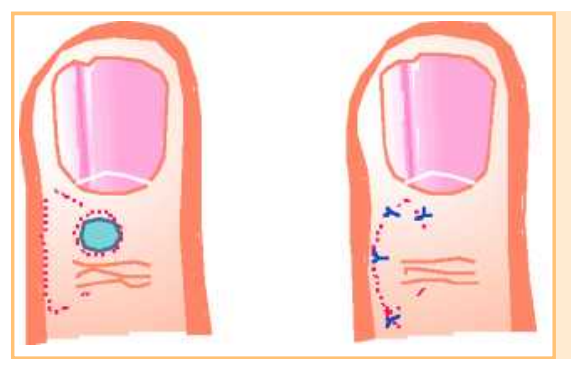

Abb. 11 Operationstechnik: Axtlappen.

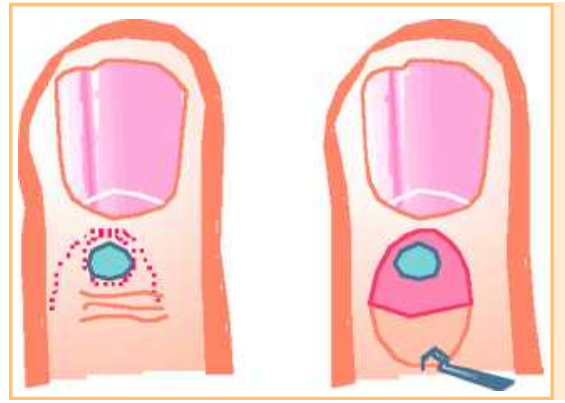

Abb. 12 Operationstechnik: einfacher Hebelappen.

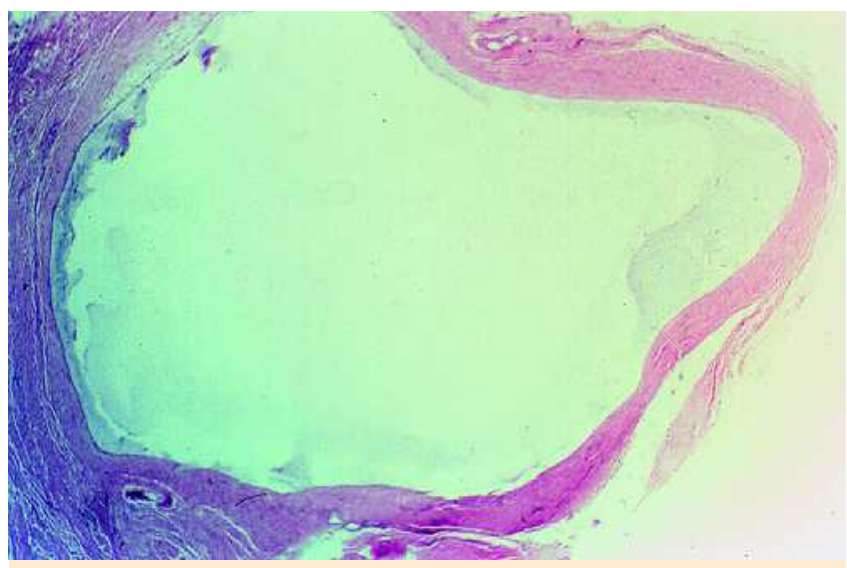

Abb. 15 Histologisches Bild der Pseudozyste.

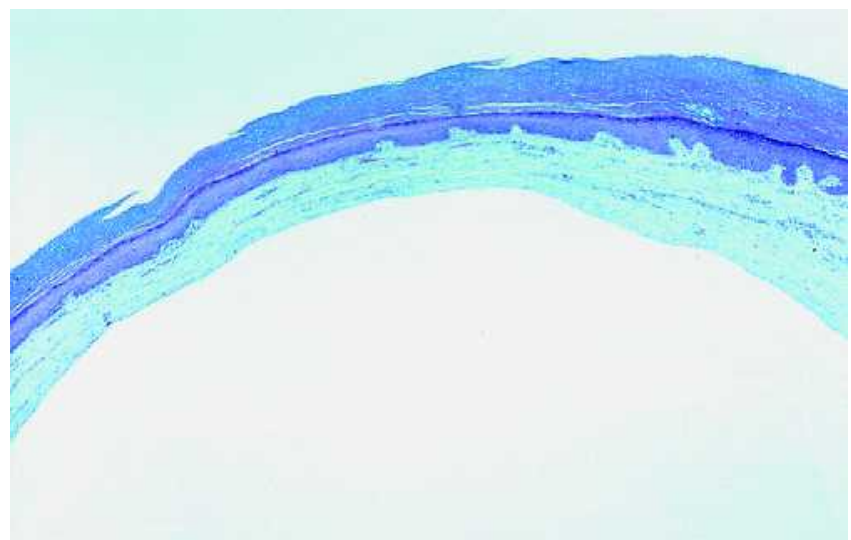

Abb. 16 Histologisches Bild der Pseudozystenwand.

Abb. 13 Operations technik: Nagelmatrixlappen.
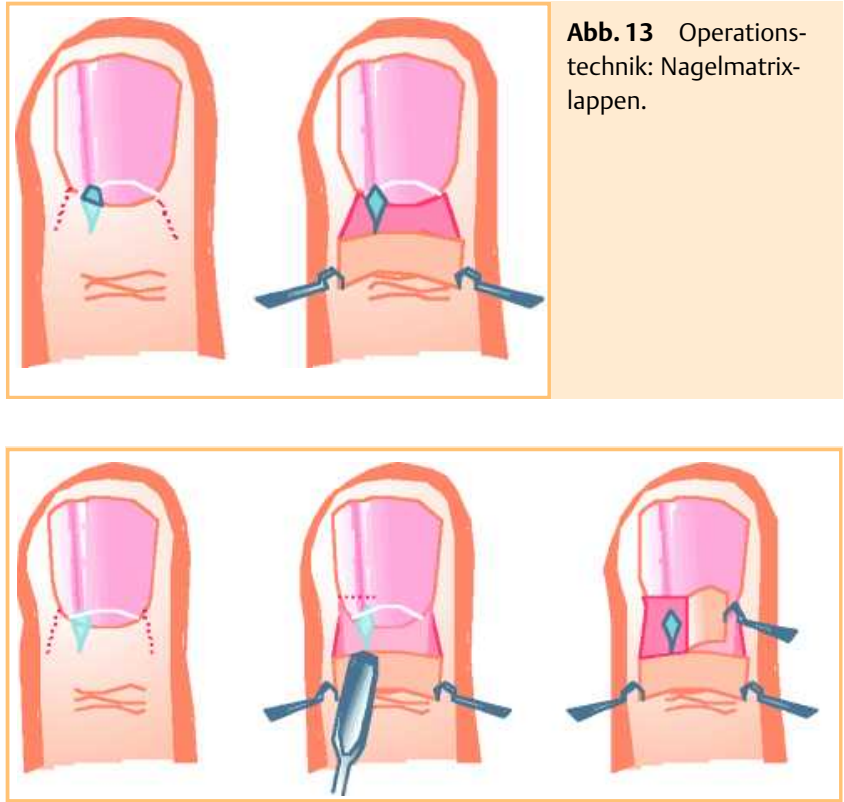

Abb. 14 Operationstechnik bei subungualer Lage.

Hämangiom, Morbus Bowen, Verruca vulgaris, Knuckle pad und Granuloma anulare zu denken. An maligne Tumoren, insbesondere ein akrolentiginöses Melanom, sollte immer gedacht und diese ggf. ausgeschlossen werden ( $\bullet$ Tab. 1) [9]. Die Diaphanoskopie, die Durchleuchtung des Fingerendglieds (Transillumina-
Tab. 1 Differenzialdiagnosen

\begin{tabular}{l} 
Verruca vulgaris \\
\hline Onychomatrikom \\
eruptives Hämangiom \\
\hline Knuckle pad \\
\hline Fibrokeratom \\
Morbus Bowen \\
Plattenepithelkarzinom \\
Glomustumor \\
Granuloma pyogenicum \\
subunguales Melanom
\end{tabular}

tion) mit einer Lichtquelle von volar und Darstellung eines opalesquen Hohlraums mit klarer Randbegrenzung, kann die Diagnose sichern [6]. Bei Verletzung der Pseudozyste entleert sich gallertartige Flüssigkeit, die der Synovia entspricht. In der feingeweblichen Untersuchung sieht man einen Hohlraum, der im Gegensatz zu einer echten Zyste nicht von Epithel ausgekleidet ist.

Pathogenetisch kommt es wahrscheinlich durch degenerative oder traumatische Schädigung des bindegewebigen Kapselapparates des Endgelenks, gleich einem Ganglion, zur Ausstülpung von Synovia, um die sich sekundär eine zarte Bindegewebskapsel bildet. Histologisch ist die Synovia als bläulich-myxoide Substanz im Pseudozystenlumen, aber auch manchmal versprengt im aufgelockerten Bindegewebe zu sehen. Nur selten gelingt histologisch die Darstellung des zum Gelenk führenden „Stiels“. 


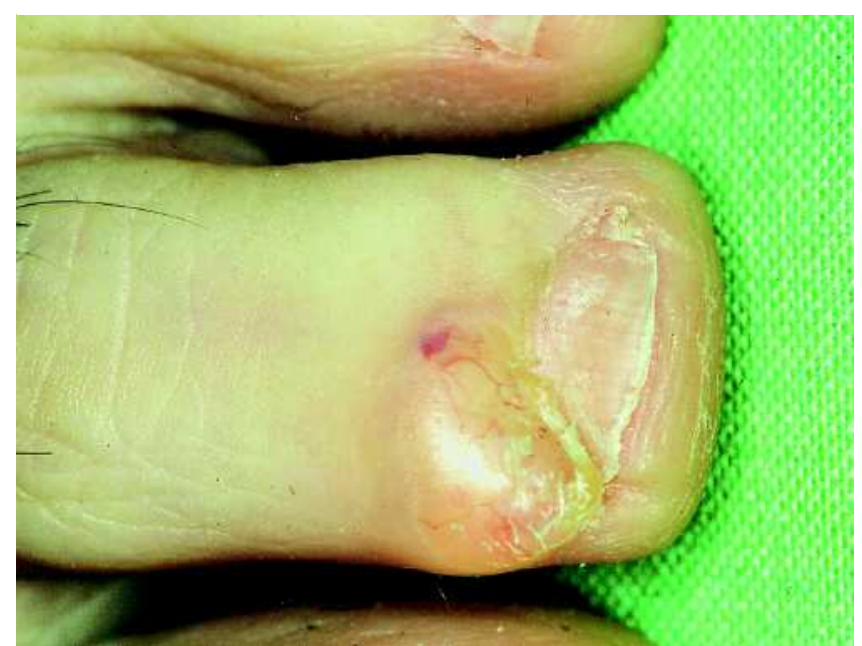

Abb. 17 Pseudozyste an der Zehe.

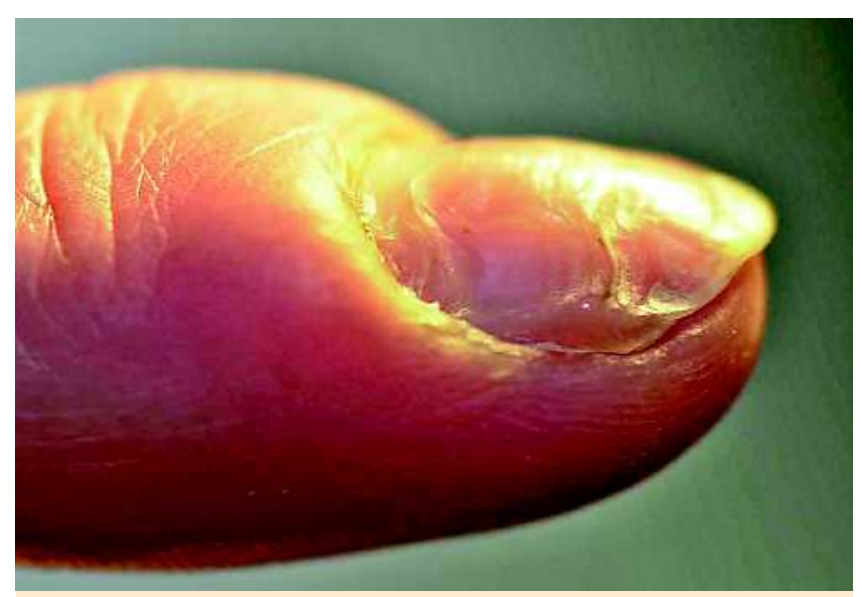

Abb. 18 Tiefe Rille im Fingernagel.

Als therapeutische Verfahren finden sich in der Literatur Hinweise auf wiederholte Punktionen und Expressionen, Injektion von Sklerosierungsmitteln (Äthoxysklerol bis zu 1\%) oder Hyaluronidase, Elektrochirurgie, chemische Ätzung mit Salpetersäure, Trichloressigsäure und Phenolum liquefactum, Kryotherapie mit flüssigem Stickstoff, $\mathrm{CO}_{2}$-Laser-Vaporisation $[1,7,8]$.

Von einer Punktion, Injektion, Kryotherapie und Lasertherapie sollte abgeraten werden, da eine Verletzung der Pseudozyste mit einem Verlust der Sterilität und daher mit einem erhöhten Infektionsrisiko für Pseudozyste und Endgelenk vergesellschaftet ist [1].

Die regelrechte Operation der myxoiden Pseudozyste, in Leitungsanästhesie nach Oberst, mit sorgfältiger Präparation, nach Anfärben mit 0,05 bis $0,1 \mathrm{ml}$ steriler Methylenblaulösung, stellt eine der sichersten, effektivsten und risikoärmsten Therapieformen dar. Als Anästhetikum hat sich bei Operationen an Fingern und Zehen Ropivacain (Naropin ${ }^{\circledR}$ ) aufgrund seiner langen post- operativen Analgesie bewährt. Die Darstellung des Stiels der myxoiden Pseudozyste zum Interphalangealgelenk und dessen Ligatur führt zu den niedrigsten Rezidivraten [3,5,6].

Als Ausnahme hiervon gilt das Vorkommen von multiplen flachen Pseudozysten, die in breiter Fläche das distale Fingerendglied bedecken und deren Exzision in einer Schwächung des Strecksehnenansatzes und einem nur schwer zu deckenden Hautdefekt resultieren würde. Hier empfiehlt sich die Injektion von z. B. Äthoxysklerol (Konzentration 0,5\%) [1].

Zusammenfassend empfehlen wir trotz des oft milden klinischen Befundes der myxoiden Pseudozyste die operative Therapie. Nach unseren Erfahrungen ist nur so eine sichere rezidivfreie Behandlung möglich, die auch Komplikationen wie Infektion der Pseudozyste mit Arthritis und drohender Endgelenksversteifung verhindert $[4,5]$.

\section{Abstract}

\section{Myxoide Pseudocyst - Diagnosis and Therapy \\ $\nabla$}

This common deformity of the nail bed is due to distal interphalangeal joint ganglions, which have been called clear cyst, mucous cyst, myxoide cyst and dorsal cyst. Pressure on the germinal matrix causes a deformed nail. When the cyst is between the dorsal skin and nail fold, pressure causes a groove in the nail. If the cyst expansion is between the periosteum and the germinal matrix it will cause destruction of the nail. The best treatment is surgery, no matter where the cyst is located.

\section{Literatur}

1 Baran R, Haneke E. Tumours of the Nail apparatus and the Adjacent Tissues. In: Baran R, Dawber R (Hrsg). Diseases of the Nails and their Management. $3^{\text {rd }}$ ed. Berlin: Blackwell, 2002: 596-601

2 Baran $R$. Finger and toe nail MRI - a useful diagnostic tool for nail tumours (free comunication). JEADV 2000; 14: 47-60

3 De Berker D, Lawrence C. Ganglion of the distal interphalangeal join (myxoid cyst) - Therapy by identification and repair of the leak of joint fluid. Arch Dermatol 2001; 137: 607-610

4 Goldman JA, Goldman L, Jaffe MS, Richfield DF. Digital mucinous pseudocysts. Arthitis and Rheumarism 1977; 20: 997-1002

5 Haneke E, Baran R, Bureau H. Chirurgie der Nagelregion. Z Hautkr 1981; 57: $1107-1116$

6 Haneke E. Operative Therapie der myxoiden Pseudozyste. In: Haneke E. (Hrsg). Fortschritte der operativen Dermatologie. Vol. 4, Gegenwärtiger Stand der operativen Dermatologie. Berlin: Springer, 1988: 231 232

7 Karrer S, Hohenleutner U, Szeimies RM et al. Treatment of digital mucous cyst with carbon dioxin laser. Acta Dermato-Venerologica 1999; 79: $224-225$

8 Kemmert D, Colver GB. Mycoid cyst treated by infrared coagulation. Clinical and Experimental Dermatologiy 1994; 19: 118-120

9 Moss SH, Schwartz KS, von Drasek-Ascher G et al. Digital venous anatomy. Journal of Hand Surgery 1985; 54A: 473-482

10 Schmoeckel C, von Mallinckrodt G, Risch M. Mukoide Dorsalzyste ganglionartige Pseudozyste des Gelenkspalts. Hautarzt 2000; 51: $682-684$ 